\title{
Research on the Characteristics and Dynamic Characteristics of Wingtip Vortex Flow Control
}

\author{
Chaoyi Wang ${ }^{1 *}$, Ze Yang ${ }^{1}$ and Junjie Zhou' \\ ${ }^{1}$ Unit95926, Aviation university of air force, Jilin Changchun 130022, China \\ * The corresponding author
}

Keywords: Wingtip vortices; Angle of attack; Aerodynamic characteristics; N-S equation

\begin{abstract}
Aircraft flying in formation in wingtip vortices influence to the lift coefficient decreases, resistance coefficient increases, lift to drag ratio decreases sharply, the stall angle of attack decreases. Correctly reflect the aircraft wing flow field around the basic information, and to reveal the variations in these performance parameters of aircraft control the extent of the impact of performance is used numerical method for aerodynamic characteristics of the aircraft and the manipulation of analysis based. In the influence of wingtip vortex numerical computing, along with the change of eddy current range, intensity, machine interference flow distribution will also change, need repeated calculation for the flow around the new changes, numerical simulation process calculation workload is huge. In this paper, based on the thorough study of Western Europe and the United States to solve the low speed viscous flow N-S equation, a set of methods based on improved SIMPLE numerical model is proposed to solve the N-S equation. This method takes into account the viscous effect of the flow field in the flight process, and has a high numerical model to solve the velocity. The results of numerical simulation of the flow field are obtained.
\end{abstract}

\section{Origin of Wing Tip Vortex}

Wing tip vortex refers to the aircraft flight maneuvers at the wing tip due to wake the phenomenon of pressure difference generated by the wing. Due to the flying wing surface of pressure difference, the airflow at the wing tip will in the pressure under the action of bottom-up flip, forming a tip as the center, backward high-speed rotating and extending downward spiral flow. Two wings of the aircraft are formed by the rotation direction of the spiral vortex outside in the opposite. Two strands of an airplane at the middle part and the formation of the updraft downdraft, and formed in the lateral wing. After the formation flying through the front vortex area, will be affected by the air flow. The formation of wingtip vortices in the plane front began to leave, until the aircraft wheel ground to achieve three roll away.

The strength of the vortex mainly by the influence of the structure of the wing tip of the plane, aircraft quality, flight speed and flight angle of attack will also have an impact on the strength, which the main is aircraft angle of attack. The vortex intensity increases with the increase of the weight of the aircraft, the decrease of the flight velocity and the increase of the angle of attack. At present, the maximum turbulent tangential velocity is $67 \mathrm{M} / \mathrm{s}$, which is measured by the experiment.

A tip vortex trends outward and downward diffusion. After the formation of the vortex will to 120 to 150 meters / minute rate decreased, in aircraft wingtip generated vortex down to 250 metres tends to level, the effect disappeared. When the crosswind flight process, vortex moves downward in under crosswind direction.

After entering the effect of eddy current zone and if the attack angle is larger, the wing surface flow separation, small aircraft will happen jitter and large aircraft will tilt to one side.

If the angle of attack is too large to stall, so when the impact of the overturning moment, the aircraft may enter the stall spiral, the aircraft may be out of control. 


\section{Wingtip Flow Field Aerodynamic Characteristics Analysis}

Pressure Correction Simple Calculation Program. Numerical simulation of flow field information needs to consider the convection term and diffusion term in the flow field, and to describe the interaction between them. Based on the above, the N-S equation is extended to the three-dimensional space to solve the flow field in the three-dimensional space, and the information of the disturbed flow field is realized. The traditional solution procedure is as follows :

The governing equations for the flow field in one dimensional space are described without considering the convection term and the diffusion term.

$$
\frac{\mathrm{d}}{\mathrm{dx}}(\rho \mathrm{u} \phi)=\frac{\mathrm{d}}{\mathrm{dx}}\left(\Gamma \frac{\mathrm{d} \phi}{\mathrm{dx}}\right)
$$

One dimensional continuity equation:

$$
\frac{\mathrm{d}}{\mathrm{dx}}(\rho \mathrm{u})=0
$$

Flow Field of Aircraft wing. For a certain type of aircraft aerodynamic characteristics and control effect of the starting point of the study is to describe the flow field, numerical flow around the wing. In the process of building a numerical model, the viscous effect can be considered, and the surface element method of classical gas dynamics can not meet the requirement of accurate calculation of flow field. In this study, the N-S equation method is used to construct the numerical model of the flow field, and the governing equations can be written in the Cartesian coordinate system:

$$
\frac{\partial \rho \phi}{\partial \mathrm{t}}+\nabla \cdot(\rho \mathrm{v} \phi-\Gamma \operatorname{grad} \phi)=\mathrm{q}
$$

Continuity equation:

$$
\phi=1, \quad \Gamma=\mu, \mathrm{q}=0
$$

$\mathrm{X}$ direction momentum equation:

$$
\phi=\mathrm{u}, \quad \Gamma=\mu, \mathrm{q}=-\frac{\partial \mathrm{P}}{\partial \mathrm{x}}+\nabla\left(\mu \frac{\partial \mathrm{v}}{\partial \mathrm{x}}\right)+\rho \mathrm{f}_{\mathrm{x}}
$$

Y direction momentum equation:

$$
\phi=\mathrm{v}, \quad \Gamma=\mu, \mathrm{q}=-\frac{\partial \mathrm{P}}{\partial \mathrm{y}}+\nabla\left(\mu \frac{\partial \mathrm{v}}{\partial \mathrm{y}}\right)+\rho \mathrm{f}_{\mathrm{y}}
$$

$\mathrm{Z}$ direction momentum equation:

$$
\phi=\mathrm{w}, \quad \Gamma=\mu, \quad \mathrm{q}=-\frac{\partial \mathrm{P}}{\partial \mathrm{z}}+\nabla\left(\mu \frac{\partial \mathrm{v}}{\partial \mathrm{z}}\right)+\rho \mathrm{f}_{\mathrm{z}}
$$

Because during actual flight of the aircraft, the wing disturbance flow is a turbulent environment, so numerical modeling process need to take into account the turbulence, this study substituted into the standard k-e turbulence model to simulate the effects of actual flight environment turbulence, the turbulent kinetic energy equation:

$$
\phi=\mathrm{k}, \quad \Gamma=\mu_{1}+\frac{\mu_{\mathrm{t}}}{\delta_{\mathrm{k}}}, \quad \mathrm{q}=\mathrm{G}_{\mathrm{k}}-\rho \varepsilon+\mathrm{G}_{\mathrm{b}}
$$


Turbulent energy dissipation rate equation:

$$
\phi=\varepsilon, \quad \Gamma=\mu_{1}+\frac{\mu_{\mathrm{t}}}{\delta_{\varepsilon}}, \quad \mathrm{q}=\frac{\varepsilon}{\mathrm{k}}\left(\mathrm{C}_{1} \mathrm{C}_{\mathrm{k}}-\mathrm{C}_{2} \rho \varepsilon\right)
$$

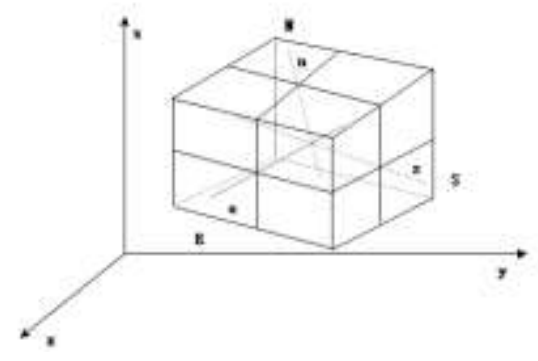

Figure 1. Finite Flow control unit

As shown in Fig. 1 of the control unit, the flow field change step integral t:

$$
\frac{1}{\Delta \mathrm{t}} \int_{\mathrm{t}}^{\mathrm{t}+\Delta \mathrm{t}} \int_{\mathrm{V}}\left[\frac{\partial \rho \phi}{\partial t}+\operatorname{div}(\rho V \phi-\Gamma g r a d \phi)\right] d V d t=\frac{1}{\Delta t} \int_{t}^{t+\Delta t} \int_{V} q d V d t
$$

In the above formula, the partial derivative term is needed to be further dispersed at the node.

To overcome this problem, in this study, application of pressure and speed relationship, through the pressure parameter correction speed and the cycle for solving the pressure equation correction equations to correct the pressure, specific procedures are as follows:

Velocity field initial value;

To solve the momentum equation, the definition of virtual speed, with human pressure equation to solve the near point speed $\mathrm{U}$.

Calculate the coefficient of the pressure equation, the solution of this equation, the flow field pressure field;

To obtain the pressure value as $\mathrm{P}^{*}, \mathrm{u}^{*}, \mathrm{v}^{*}$ into the momentum equation, $\mathrm{w}^{*}$;

Calculation of the quality of the source $\mathrm{B}$, the pressure correction equation;

Into the momentum correction velocity field;

The pressure parameter correction velocity, and the desired speed re substituted into the discrete momentum equation, is solved to obtain the pressure value, the pressure value iteration to a new round of computation. The iterative convergence of the judgment equation or not, if the convergence, then completed the calculation; otherwise, return to the second step to continue the iterative solution.

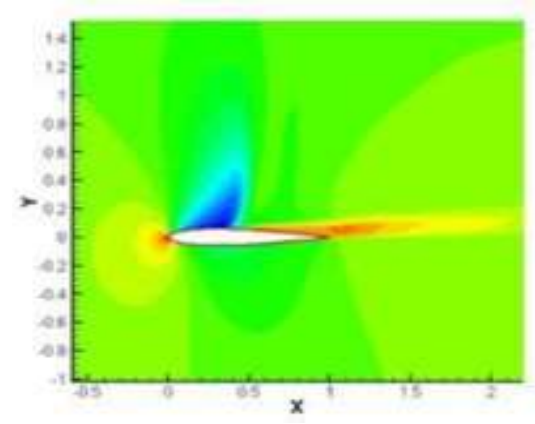

Figure 2. Finite The flow field information of a certain type of aircraft wing

In summary, the flow field around the airfoil can be obtained by solving the discrete equations by solving the algebraic equations. The flow field information of a certain type of aircraft wing is shown in Fig. 2. 
Accompanied by wingtip vortices, the machine in motion process has a slight jitter phenomenon, which is due to the eddy current flow direction on the machine under the pressure function, then in the front position of the vortex, the roll after the captain; in addition, because of the vortex flow trajectories in the horizontal plane was s shape, so on machine milling effect also showed to center deviation phenomenon. When the distance reaches a certain degree, the wingtip vortex aerodynamic effect will be very attenuation. The wingtip vortex aerodynamic effect is as Fig. 3 to Fig. 4.
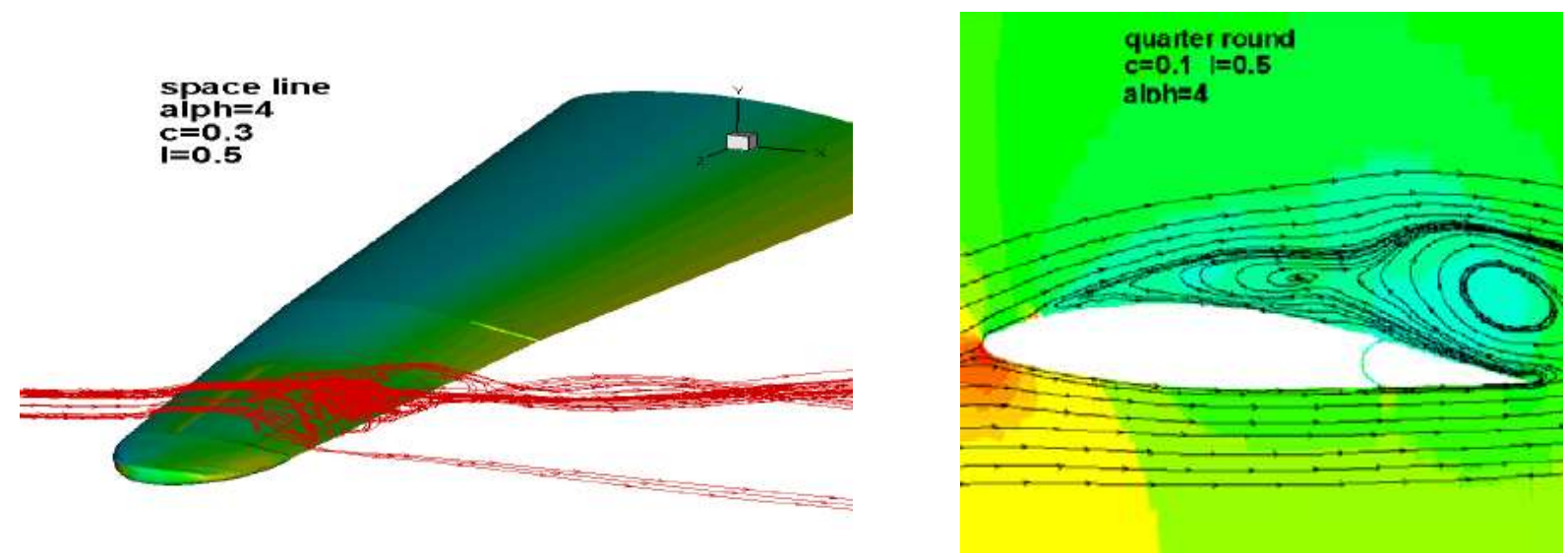

Figure 3. Finite Three dimensional simulation Figure 4. Finite Two dimensional simulation

\section{Conclusion}

This research mainly carried on the following work:

On the basis of studying the existing theory of computational fluid dynamics, the N-S equations are solved to solve the flow field around the aircraft;

Using ANSYS finite element analysis software to analyze the aerodynamic characteristics of aircraft;

The numerical simulation system of aircraft aerodynamic characteristics and control is studied. For understanding the gas dynamic torque in the control of the process of the aircraft impact, and reveal the operation process of the aerodynamic force on the flight of. It is conducive to improving the pilot to grasp the control law under different aerodynamic conditions, which is beneficial for the pilot to grasp the limit performance of the aircraft aerodynamic; it is beneficial to provide theoretical basis for the formulation of the operational norms. In order to solve the influence of different weather conditions on the negative aerodynamic torque of pilot operated aircraft, the theoretical basis is provided for solving the aerodynamic effect of the formation flying.

\section{References}

[1] Yi Xian, Iced airfoil numerical simulation and iced airfoil of gas dynamic characteristics analysis [D]. Beijing: China Aerodynamics Research and Development Center (doctoral dissertation, 2002:35-42.

[2] Li Shumin, low speed viscous flow calculation method and Application Research [D]. China Aerodynamics Research and development center doctoral dissertation, 2004:16-22.

[3] Li Shumin, Zhu Guolin, Wang Kaichun. Parallel implementation of two-dimensional incompressible N-S equations based on partition [J]. Journal of aerodynamics, 2002,20:23-24.

[4] Guo Bin, Xiong ray, Chen Xiaobo, et al. Research and implementation of universal adapter MATLAB and [J]. HLA/RTI Journal of system simulation, 2004, 16 (6): 1275-1279. 